\title{
Vital Signs Sponsor Defined Identifier
}

National Cancer Institute

\section{Source}

National Cancer Institute. Vital Signs Sponsor Defined Identifier. NCI Thesaurus. Code C83084.

One or more sponsor-defined characters used to identify, name, or characterize the vital signs test. 